\title{
Multichannel Sampling of Multidimensional Parametric Signals
}

\author{
Hojjat Akhondi Asl \\ Pier Luigi Dragotti \\ CSP Group, Electrical and Electronic Engineering Department, \\ Imperial College London, Exhibition Road, SW7 2AZ, England. \\ \{hojjat.akhondi-as103, P.Dragotti\}@imperial.ac.uk *
}

\begin{abstract}
The contribution of this paper is two-fold; first, we present a novel approach for sampling and reconstructing two dimensional signals with parametric structure or namely signals with Finite Rate of Innovation (FRI). The considered signals are bi-level polygons and set of 2-D Diracs and it will be shown that with the use of ACMP method, projection-slice theorem, Radon projections and exponential splines as sampling kernels, such signals can be perfectly reconstructed. Then, we present a possible extension of the theory of sampling multidimensional FRI signals, discussed above, to the case of multichannel acquisition systems. The essential issue of our considered multichannel system is that each channel receives the input signal with an unknown geometric transformation which needs to be estimated. We pose both the synchronization stage and the signal reconstruction stage as a parametric estimation problem and demonstrate that a simultaneous exact synchronization of the channels and reconstruction of the FRI signal is possible.
\end{abstract}

Key words and phrases : Multichannel sampling, Multidimensional FRI signals, Exponential splines.

2000 AMS Mathematics Subject Classification - 94A20, 65D07

\section{Introduction}

Sampling is the process in which a continuous-time signal $g(x)$ is represented by a discrete set of values or samples $g[n]$, where $n \in \mathbb{Z}$. The fundamental questions of interest for such a process are, 1) Under what conditions signal $g(x)$ is perfectly and uniquely recovered from the set of samples $g[n]$ and 2) What are the methods of reconstruction? The classical answer to these key questions was given by Shannon in his well-known sampling theorem, which states that any bandlimited continuous-time signal $g(x)$ can be sampled and perfectly reconstructed if the

${ }^{*}$ This work was in part presented at ICASSP09 [2] and SAMPTA09 [1]. 
sampling rate is chosen to be equal or greater than twice the maximum nonzero frequency of the signal. The reconstruction of the original signal from its samples is then obtained using a sinc interpolation function. This extremely fruitful result however, has two major drawbacks. First, real world signals are never exactly bandlimited and second, an ideal sinc interpolation function is physically not implementable. Thus, different approximations need to be taken into account for such a sampling scheme to work in practice. These limitations have led researchers to re-examine some of the core ideas of Shannon's theory and take it further into more advanced sampling techniques.

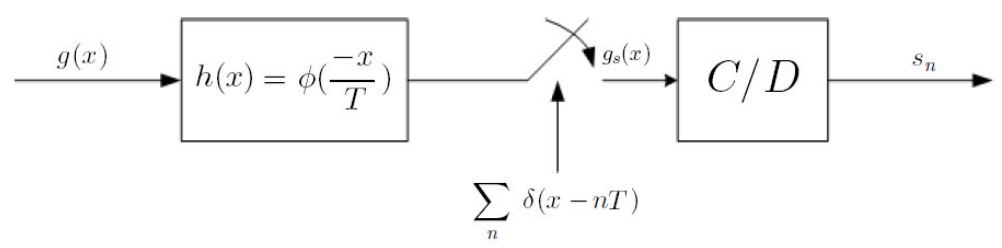

Figure 1: Sampling set-up. Here, $g(x)$ is the continuous-time signal, $h(x)$ the impulse response of the acquisition device and $T$ the sampling period. The measured samples are $s_{n}=\langle g(x), \phi(x / T-n)\rangle$.

Recently in $[7,6,26]$, it has been shown that it is possible to sample and perfectly reconstruct some classes of non-bandlimited signals. In these schemes, the prior that the signal is sparse in a basis or in a parametric space is taken into account and perfect reconstruction is achieved based on a set of suitable measurements. Depending on the set-up used and reconstruction method involved, these sampling methods go under different names such as compressed sensing (CS), compressive sampling [7,6] or sampling signals with finite rate of innovation (FRI) $[26,8]$. Signals that can be sampled with the latter framework, shown in Figure 1, include streams of Diracs, piecewise-polynomial $[26,8]$ and piecewise-sinusoidal signals [5]. The reconstruction of these FRI signals is based on the annihilating filter method (also known as Prony's method [22]) and the reader can refer to Appendix A for a brief overview of the method.

Most of these sampling schemes focus on a single-channel acquisition model, however, modern and fast Analogue-to-Digital Converters (ADC) and majority of sensor networks use interleaved multichannel converters which allow a reduction in the complexity of the devices while keeping higher rates of conversion. Given the practical importance of multichannel acquisition devices, it is natural to investigate extensions of sparse sampling theories to the multichannel scenario. The critical issue in multichannel sampling, shown in Figure 2, is the precise synchronization of the various channels, since different devices introduce different drifts and different gains (due to imperfections of electronic circuits 


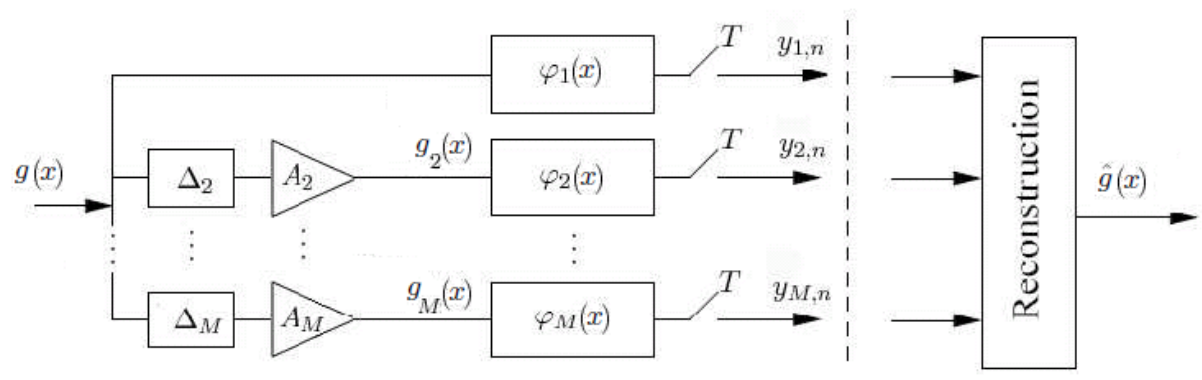

Figure 2: Multichannel sampling set-up. Here, the continuous-time signal $g(x)$ is received by multiple channels with multiple acquisition devices. The samples $y_{i, n}$ for $i=1,2, \ldots, M$, from each channel are utilized jointly for the reconstruction process. The parameters $\Delta_{i}$ and $A_{i}$ for $i=2,3, \ldots, M$ represent the different delay and gain parameters introduced within the channels.

for example) that need to be estimated together with the signal itself. In this paper we will be considering the multichannel sampling of multidimensional FRI signals and extend the results in $[26,8]$ to this new scenario.

The paper is organized as follows: In the next section, we will briefly present the sampling setup required for sampling 2-D FRI signals and also discuss the properties of the sampling kernels involved. In Section 3 we will introduce our algorithms for sampling set of 2-D Diracs and bi-level polygons and in Section 4 we will describe our method for sampling such signals in a multichannel framework. We finally conclude in Section 5.

\section{Sampling Setup for Multidimensional Parametric Signals}

The problem of sampling two dimensional signals is more involved and does not allow direct extension of the 1-D results (see Section 3.1). Recently, extensions to the multidimensional case were considered by Maravic et al. [17] and Shukla et al. [21]. Maravic et al. considered 2-D FRI signals, such as 2-D set of Diracs and bi-level polygons and used the sinc and Gaussian sampling kernels. Shukla et al. proposed algorithms, from the theory of complex moments, for sampling the same 2-D signals but with the use of B-splines as the sampling kernel. The sinc and the Gaussian sampling kernels have infinite support and are not physically realizable and also such kernels make the reconstruction algorithm unstable. In this paper we will be considering exponential reproducing kernels and in particular exponential splines [23], since they benefit from having compact support and from being practically implementable ( $\mathrm{RC}$ circuits for example). E- 
splines also tend to be more stable than other kernels.

Figure 3 shows the sampling setup used for sampling 2-D FRI signals. From the setup shown, the samples $s_{j, k}$ are given by:

$$
s_{j, k}=\int_{-\infty}^{\infty} \int_{-\infty}^{\infty} g(x, y) \phi\left(\frac{x}{T_{x}}-j, \frac{y}{T_{y}}-k\right) d x d y,
$$

where the kernel $\phi(x, y)$ is the time reversed version of the filter response and is assumed to be given by the tensor product of two 1-D functions, that is $\phi(x, y)=\phi(x) \phi(y)$.

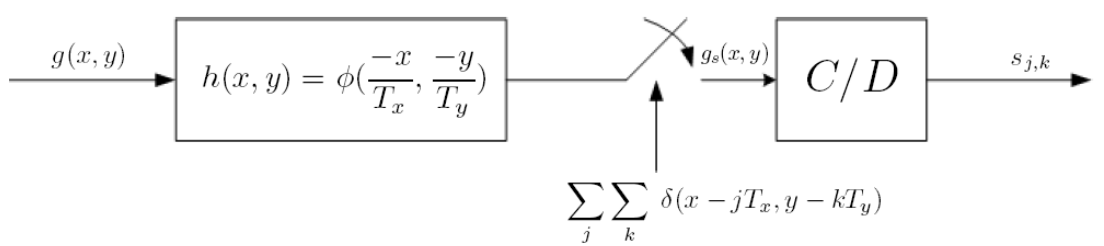

Figure 3: 2-D sampling setup. Here, $g(x, y)$ represents the input FRI signal, $\phi(x, y)$ the sampling kernel, $g_{s}(x, y)$ the sampled version of the input signal $g(x, y), s_{j, k}$ the samples and $T_{x}, T_{y}$ are the sampling intervals along the horizontal and vertical directions respectively.

As mentioned before, in this paper we will focus on a specific class of kernels that are able to reproduce real or complex exponentials. A kernel $\phi(x, y)$ is able to reproduce exponentials up to order $M$ and $N$ along the $x$ and $y$ axes respectively, if there exists coefficients $c_{j, k}^{m, n} \in \mathbb{C}$ such that:

$$
\sum_{j \in \mathbb{Z}} \sum_{k \in \mathbb{Z}} c_{j, k}^{m, n} \phi(x-j, y-k)=e^{\alpha_{m} x} e^{\beta_{n} y}, \quad \alpha_{m}, \beta_{n} \in \mathbb{C},
$$

where $m=0,1, \ldots, M$ and $n=0,1, \ldots, N$. The coefficients $c_{j, k}^{m, n}$ can be found numerically and the choice of the exponents is restricted to $\alpha_{m}=\alpha_{0}+m \lambda_{1}$ and $\beta_{n}=\beta_{0}+n \lambda_{2}$. This is done to allow the use of specific reconstruction techniques which are described later on.

Exponential splines (E-splines) [23] are central to the exponential reproduction theory and a function $\beta_{\vec{\alpha}, \vec{\beta}}(x, y)$ with Fourier transform:

$$
\hat{\beta}_{\vec{\alpha}, \vec{\beta}}\left(\omega_{1}, \omega_{2}\right)=\prod_{m=0}^{M} \prod_{n=0}^{N}\left(\frac{1-e^{\alpha_{m}-j \omega_{1}}}{j \omega_{1}-\alpha_{m}}\right)\left(\frac{1-e^{\beta_{n}-j \omega_{2}}}{j \omega_{2}-\beta_{n}}\right),
$$

is called an E-spline of order $(M+1) \times(N+1)$ where $\vec{\alpha}=\left(\alpha_{0}, \alpha_{1}, \ldots, \alpha_{M}\right)$ and $\vec{\beta}=\left(\beta_{0}, \beta_{1}, \ldots, \beta_{N}\right)$ can be real or complex. The resulting spline has compact support and can reproduce any exponential in the subspace spanned 
by $\left\{e^{\alpha_{0} x} e^{\beta_{0} y}, e^{\alpha_{1} x} e^{\beta_{0} y}, \ldots, e^{\alpha_{M} x} e^{\beta_{N} y}\right\}$. In time-domain, the expression of a 1-D E-spline of order one is given by:

$$
\beta_{\alpha_{0}}(x)= \begin{cases}e^{\alpha_{0} x} & 0 \leq x<1 \\ 0 & \text { otherwise }\end{cases}
$$

where the higher order E-splines are obtained by successive convolutions of lower order ones ((M+1)-fold convolution).

As mentioned above, the two-dimensional E-spline kernel can be obtained by the tensor product between two single-dimension splines. It is interesting to point out that, since the exponential reproduction formula is preserved through convolution [23], any composite function of the form $\phi(x) * \beta_{\vec{\alpha}_{M}}(x)$ is also able to reproduce exponentials. Another interesting result which can be obtained from the above sampling setup is that, if we denote $\tau_{m, n}$ as follows

$$
\tau_{m, n}=\sum_{j} \sum_{k} c_{j, k}^{m, n} s_{j, k},
$$

then by expanding the samples $s_{j, k}$ and replacing the corresponding equations (assuming $T_{x}=T_{y}=1$ for simplicity), the exponential moments of the signal $g(x, y)$ are obtained, that is:

$$
\begin{aligned}
\tau_{m, n} & =\left\langle g(x, y), \sum_{j} \sum_{k} c_{j, k}^{m, n} \phi(x-j, y-k)\right\rangle \\
& =\left\langle g(x, y), e^{\alpha_{m} x} e^{\beta_{n} y}\right\rangle \\
& =\int_{-\infty}^{\infty} \int_{-\infty}^{\infty} g(x, y) e^{\alpha_{m} x} e^{\beta_{n} y} d x d y .
\end{aligned}
$$

In the case of purely imaginary exponentials, that is setting $\alpha_{m}$ and $\beta_{n}$ to be purely imaginary, $\tau_{m, n}$ correspond to the Fourier transform of $g(x, y)$ evaluated at $\left(\alpha_{m}, \beta_{n}\right)$, that is:

$$
\tau_{m, n}=G\left(\alpha_{m}, \beta_{n}\right),
$$

where $G(u, v)$ represents the Fourier transform of the signal $g(x, y)$. Having gone through the basics needed for sampling multidimensional FRI signals, we will now propose our algorithms for sampling and perfectly reconstructing set of 2-D Diracs and bi-level polygons using E-splines.

\section{A Sampling Theorem for Multidimensional Para- metric Signals}

In this section we present our novel algorithms for sampling and perfectly reconstructing 2-D set of Diracs and bi-level polygons using E-spline sampling kernels. 


\subsection{A Sampling Theorem for Set of 2-D Diracs}

Let us assume that a set of 2-D Diracs is passed through the sampling setup shown in Figure 3. Assuming that there are $K$ Diracs in the signal, such a signal can be represented as:

$$
g(x, y)=\sum_{k=1}^{K} a_{k} \delta\left(x-x_{k}, y-y_{k}\right),
$$

where $a_{k}$ are the amplitudes and $\left(x_{k}, y_{k}\right)$ are the horizontal and vertical coordinates of the Diracs respectively. Since each Dirac has an amplitude and also a horizontal and vertical location, the signal has $3 K$ degrees of freedom. Now by substituting Equation (9) in (4) and (7), we obtain:

$$
\begin{aligned}
\tau_{m, n} & =\sum_{j} \sum_{k} c_{j, k}^{m, n} s_{j, k} \\
& =\int_{-\infty}^{\infty} \int_{-\infty}^{\infty} g(x, y) e^{\alpha_{m} x} e^{\beta_{n} y} d x d y \\
& =\sum_{k=1}^{K} a_{k} \int_{-\infty}^{\infty} \int_{-\infty}^{\infty} \delta\left(x-x_{k}, y-y_{k}\right) e^{\alpha_{m} x} e^{\beta_{n} y} d x d y \\
& =\sum_{k=1}^{K} a_{k} e^{\alpha_{m} x_{k}} e^{\beta_{n} y_{k}} .
\end{aligned}
$$

Therefore, given the samples $s_{j, k}$ we can obtain $\tau_{m, n}=\sum_{k=1}^{K} a_{k} e^{\alpha_{m} x_{k}} e^{\beta_{n} y_{k}}$ and the aim now is to estimate the parameters $\left(a_{k}, x_{k}, y_{k}\right)$ from $\tau_{m, n}$. One might suggest an extension of the annihilating filter method described in $[26,8]$ for $1-\mathrm{D}$ signals, to this scenario. This extension fails because the relation $\tau_{m, n} * h_{m, n}=0$, where $h_{m, n}$ is the annihilating filter, has an infinite number of zeros over the complex field and there exists no unique solution for such a problem.

Another way to tackle this problem is by setting the indices $m$ and $n$ to zero one at time and applying the 1-D annihilating filter method on both sets to find the values of $x_{k}$ and $y_{k}$ coordinates separately. There are two drawbacks with this method: first, the estimated locations have to be paired and this is a combinatorial problem which may not have a unique solution, namely two different pairings may lead to the same samples $s_{j, k}$ and second, in the case of having common coordinates between $x_{k}$ and $y_{k}$, the annihilating filter method is unable to find the multiple poles, because of having non-unique filter coefficients.

This is indeed a spectral estimation problem and among the earliest techniques that addressed this problem was the matrix enhancement and matrix pencil (MEMP) algorithm by Hua [12]. For the case of common coordinates problem, Hua solves the rank deficiency problem by introducing an enhanced 
matrix of the original data matrix. In this way, a partitioned and stacked Hankel matrix of the original data matrix is constructed in a way such that the full-rank property of the original matrix is restored. For the pairing problem, an unattractive combinatorial approach is suggested, trying all the possible combinations to find the correct pairing and this is computationally expensive and therefore not efficient. The ACMP (Algebraically Coupled Matrix Pencils) method by F. Vanpoucke et al. [25] however, introduces a new technique to find the correct pairings by simultaneously solving two algebraically related generalized eigenvalue equations. As the matrix enhancement approach in [12] is not compatible with the algebraic pairing technique, an alternative rank restoration technique is introduced. We will now briefly outline the ACMP algorithm, but more detailed discussions can be found in [25].

\subsubsection{Outline of the ACMP Method}

Given $\alpha_{m}=\alpha_{0}+m \lambda_{1}$ and $\beta_{n}=\beta_{0}+n \lambda_{2}$, the obtained measurements $\tau_{m, n}$, which consists of a sum of $K$ exponentials (complex or real) with unknown coordinate pairs $x_{k}$ and $y_{k}$, and amplitudes $a_{k}$, can be rewritten as:

$$
\tau_{m, n}=\sum_{k=1}^{K} a_{k} e^{\alpha_{m} x_{k}} e^{\beta_{n} y_{k}}=\sum_{k=1}^{K} \widehat{a_{k}} \varphi_{k}^{m} \psi_{k}^{n},
$$

where $\widehat{a_{k}}=a_{k} e^{\alpha_{0} x_{k}} e^{\beta_{0} y_{k}}, \varphi_{k}=e^{\lambda_{1} x_{k}}$ and $\psi_{k}=e^{\lambda_{2} y_{k}}$. Let the $K(K+1) \times K(K+$ 1) enhanced matrix $J$ be defined as:

$$
J=\left[\begin{array}{cccc}
H^{(1,1)} & H^{(2,1)} & \ldots & H^{(K, 1)} \\
H^{(1,2)} & H^{(2,2)} & \ldots & H^{(K, 2)} \\
\vdots & \vdots & \ddots & \vdots \\
H^{(1, K)} & H^{(2, K)} & \ldots & H^{(K, K)}
\end{array}\right]
$$

where each block matrix $H^{(l, k)}$ of size $K \times K$ is given by:

$$
H^{(l, k)}=\tau_{l: K+l, k: K+k}
$$

For the construction of matrix $J$, at least $2 K \times 2 K$ data points are required. This new enhanced matrix can be decomposed as follows:

$$
J=X^{\prime} A Y^{\prime T},
$$

where

$$
\begin{gathered}
X^{\prime}=\left[\begin{array}{lllll}
X_{K+1}^{T} & \Psi X_{K+1}^{T} & \Psi^{2} X_{K+1}^{T} & \ldots & \Psi^{K-1} X_{K+1}^{T}
\end{array}\right]^{T} \\
Y^{\prime}=\left[\begin{array}{lllll}
Y_{K+1}^{T} & \Phi Y_{K+1}^{T} & \Phi^{2} Y_{K+1}^{T} & \ldots & \Phi^{K-1} Y_{K+1}^{T}
\end{array}\right]^{T} .
\end{gathered}
$$


Here, the matrices $X^{\prime}$ and $Y^{\prime}$ are both of sizes $K(K+1) \times K$. Furthermore, $\Phi, \Psi$ and $A$ are $K \times K$ diagonal matrices with parameters $\left\{\varphi_{1}, \varphi_{2}, \ldots, \varphi_{K}\right\}$, $\left\{\psi_{1}, \psi_{2}, \ldots, \psi_{K}\right\}$ and $\left\{\widehat{a_{1}}, \widehat{a_{2}}, \ldots, \widehat{a_{K}}\right\}$ along the diagonals respectively. The matrices $X_{K+1}$ and $Y_{K+1}$ are given by:

$$
X_{K+1}=\left[\begin{array}{cccc}
1 & 1 & \ldots & 1 \\
\varphi_{1} & \varphi_{2} & \ldots & \varphi_{K} \\
\vdots & \vdots & \ddots & \vdots \\
\varphi_{1}^{K} & \varphi_{2}^{K} & \ldots & \varphi_{K}^{K}
\end{array}\right] \quad Y_{K+1}=\left[\begin{array}{cccc}
1 & 1 & \ldots & 1 \\
\psi_{1} & \psi_{2} & \ldots & \psi_{K} \\
\vdots & \vdots & \ddots & \vdots \\
\psi_{1}^{K} & \psi_{2}^{K} & \ldots & \psi_{K}^{K}
\end{array}\right] .
$$

The matrices $X^{\prime}$ and $Y^{\prime}$ have a Vandermonde structure and when the matrix $J$ is at least of size $K(K+1) \times K(K+1)$, they are both full-rank . This matrix enhancement technique restores the full-rank property of the original matrix for the case of common coordinates problem. Now, due to the Vandermonde structure of matrices $Y^{\prime}$ and $X^{\prime}$, the four sub-matrices $J_{t l}, J_{t r}, J_{b l}$ and $J_{b r}$ of matrix $J$ are constructed, which correspond to the omission of the first and last rows and columns on each block of the matrix $J$ :

$$
\begin{aligned}
& J_{t l}=\underline{X^{\prime}} A{\underline{Y^{\prime}}}^{T} \\
& J_{t r}=\underline{X^{\prime}} A \overline{Y^{\prime}}=\underline{X^{\prime}} A \Psi \underline{Y^{\prime}} \\
& J_{b l}=\overline{X^{\prime}} A \underline{Y^{\prime}}=\underline{X^{\prime}} \Phi A \underline{Y^{\prime T}} \\
& J_{b r}=\overline{X^{\prime} A \overline{Y^{\prime}}}=\underline{X^{\prime}} \Phi A \Psi{\underline{Y^{\prime}}}^{T} .
\end{aligned}
$$

Here, $\overline{X^{\prime}}$ and $X^{\prime}$ indicate the omission of the first and last row of matrix $X^{\prime}$ respectively. From the matrices described above we can obtain two matrix pencils $J_{t r}-\mu J_{t l}$ and $J_{b l}-\lambda J_{t l}$. The ACMP method then operates as follows: First the SVD of $J_{t l}$ is computed as follows:

$$
J_{t l}=U \Sigma V^{H},
$$

where $H$ is Hermitian operator. By multiplying $U^{H}$ to the left hand side and multiplying $V$ to the right hand side of the two matrix pencils defined above we obtain:

$$
\begin{aligned}
U^{H}\left(J_{t r}-\mu J_{t l}\right) V & =U^{H} \underline{X^{\prime}} A \Psi \underline{Y^{\prime T}} V-\mu U^{H} \underline{X^{\prime}} A{\underline{Y^{\prime T}}}^{T} V \\
& =F \Psi G-\mu F G \\
& =C_{t r}-\mu C_{t l},
\end{aligned}
$$

and,

$$
\begin{aligned}
U^{H}\left(J_{b l}-\lambda J_{t l}\right) V & =U^{H} \underline{X^{\prime}} A \Phi \underline{Y^{\prime T}} V-\lambda U^{H} \underline{X^{\prime}} A \underline{Y^{\prime T}} V \\
& =F \Phi G-\lambda F G \\
& =C_{b l}-\lambda C_{t l},
\end{aligned}
$$


where $F=U^{H} \underline{X^{\prime}} A, G=\underline{Y^{\prime T}} V, C_{t r}=U^{H} J_{t r} V=F \Psi G, C_{b l}=U^{H} J_{b l} V=F \Phi G$ and $C_{t l}=U^{H} J_{t l} V=F G=\Sigma$. By applying Eigen-Value-Decomposition (EVD) on the new matrix pencils $C_{t r}-\mu C_{t l}$ and $C_{b l}-\lambda C_{t l}$, each of the poles $\varphi_{k}$ and $\psi_{k}$ are obtained:

$$
\begin{aligned}
& \operatorname{eig}\left(C_{t l}^{-1} C_{t r}\right)=\operatorname{eig}\left(G^{-1} F^{-1} F \Psi G\right)=\operatorname{eig}\left(G^{-1} \Psi G\right)=\Psi \\
& \operatorname{eig}\left(C_{t l}^{-1} C_{b l}\right)=\operatorname{eig}\left(G^{-1} F^{-1} F \Phi G\right)=\operatorname{eig}\left(G^{-1} \Phi G\right)=\Phi .
\end{aligned}
$$

The identical transformation $G$ on both equations guarantees that we have the correct pairing for the estimated $\varphi_{k}$ and $\psi_{k}$ values. The steps below present a pseudo code of the algorithm:

1. Construct the enhanced matrix $J$ from the moments.

2. Construct the sub-matrices $J_{t l}, J_{t r}$ and $J_{b l}$ from the matrix $J$.

3. Compute the singular value decomposition (SVD) of the sub-matrix $J_{t l}$, that is $J_{t l}=U \Sigma V^{H}$.

4. Generate the matrices $C_{t l}, C_{b l}$ and $C_{t r}$ with the following equations: $C_{t l}=$ $\Sigma, C_{t r}=U^{H} J_{t r} V, C_{b l}=U^{H} J_{b l} V$.

5. Apply the Eigen-Value-Decomposition to $C_{t l}^{-1} C_{t r}$ and $C_{t l}^{-1} C_{b l}$. This leads to the unknown poles since: $\operatorname{eig}\left(C_{t l}^{-1} C_{t r}\right)=\Psi$ and $e i g\left(C_{t l}^{-1} C_{b l}\right)=\Phi$.

As the exact values of the poles $\varphi_{k}$ and $\psi_{k}$ are found using the above method, the matrices $X^{\prime}$ and $Y^{\prime}$ can be constructed to obtain the parameters $\widehat{a_{k}}$, using the following equation:

$$
A=\left(X^{\prime \dagger}\right) J\left(Y^{\prime T}\right)^{\dagger}
$$

where $\dagger$ stands for pseudo-inverse. From the estimated parameters $\widehat{a_{k}}$ and the poles $\varphi_{k}$ and $\psi_{k}$ we can easily find the amplitudes $a_{k}$ and coordinates $x_{k}$ and $y_{k}$ as follows:

$$
x_{k}=\frac{\ln \left(\phi_{k}\right)}{\lambda_{1}}, \quad y_{k}=\frac{\ln \left(\psi_{k}\right)}{\lambda_{2}}, \quad a_{k}=\frac{\widehat{a_{k}}}{e^{\alpha_{0} x_{k}} e^{\beta_{0} y_{k}}} .
$$

As mentioned above, for a set of $K$ 2-D Diracs, at least $2 K \times 2 K$ data points are required for the construction of the enhanced matrix. This means that the 2-D exponential spline order need to be at least $(2 K \times 2 K)$ in order to reproduce the $2 K$ exponential moments along both $x$ and $y$ axes. Regarding the number of samples required, it was shown in [8] for 1-D signals that if there are no more than K Diracs in an interval of size $\tau=2 K L T$, L denoting the support of the sampling kernel, then we are guaranteed that two groups of $\mathrm{K}$ consecutive Diracs are sufficiently distant and that they are separated by some zero samples. By locating these zeros, one can separate the two groups and 
apply the corresponding reconstruction method on each group independently. Thus, the minimum number of samples required to perfectly reconstruct $\mathrm{K} 2$-D Diracs is $N=2 K L$ along both Cartesian axes. We can now summarize the above discussion with the following proposition:

Proposition I - A set of $K$ 2-D Diracs is uniquely determined from the samples $s_{j, k}=\left\langle g(x, y), \phi\left(\frac{x}{T_{x}}-j, \frac{y}{T_{y}}-k\right)\right\rangle$ with a minimum number of samples $N=2 K L$ along both the Cartesian axes $x$ and $y$, provided that the sampling kernel $\phi(x, y)$ with support $L$, can reproduce exponentials with an order $2 K$ along both the Cartesian axes.

Having presented a sampling theorem for set of 2-D Diracs, we now move on to our next input signal, Bi-level Polygons.

\subsection{A Sampling Theorem for Bi-level Polygons}

Consider a non-intersecting, convex and bi-level $K$-sided polygon with vertices at points $\left(x_{k}, y_{k}\right), k=1,2, \ldots, K$. The described polygon can be uniquely specified by its $K$ vertices and therefore has degrees of freedom equal to $2 K$. Lee and Mittra [14] derived a general formula for the Fourier transform of any K-sided bi-level polygon where they showed that the Fourier transform is directly related to the location of the polygon's vertices $\left(x_{k}, y_{k}\right)$ :

$$
G(u, v)=\sum_{k=1}^{K} e^{j\left(u x_{k}+v y_{k}\right)} \frac{p_{k-1}-p_{k}}{\left(u+p_{k-1} v\right)\left(u+p_{k} v\right)} .
$$

Here, $p_{k}$ represent the gradients of the polygonal lines. The reader can refer to [14] for the derivation of this result. As $\tau_{m, n}$ are the exponential moments of the input signal and given $\alpha_{m}=\alpha_{0}+m \lambda_{1}$ and $\beta_{n}=\beta_{0}+n \lambda_{2}$, we can deduce the following:

$$
\begin{aligned}
\tau_{m, n} & =G\left(\alpha_{m}, \beta_{n}\right) \\
& =\sum_{k=1}^{K} e^{\left(\alpha_{m} x_{k}+\beta_{n} y_{k}\right)} \frac{p_{k-1}-p_{k}}{\left(\alpha_{m}+p_{k-1} \beta_{n}\right)\left(\alpha_{m}+p_{k} \beta_{n}\right)} \\
& =\sum_{k=1}^{K} a_{k, m, n} e^{\alpha_{m} x_{k}} e^{\beta_{n} y_{k}} \\
& =\sum_{k=1}^{K} \widehat{a}_{k, m, n} \varphi_{k}^{m} \psi_{k}^{n},
\end{aligned}
$$

where $a_{k, m, n}=\frac{p_{k-1}-p_{k}}{\left(\alpha_{m}+p_{k-1} \beta_{n}\right)\left(\alpha_{m}+p_{k} \beta_{n}\right)}, \widehat{a}_{k, m, n}=a_{k, m, n} e^{\alpha_{0} x_{k}} e^{\beta_{0} y_{k}}, \varphi_{k}=e^{\lambda_{1} x_{k}}$ and $\psi_{k}=e^{\lambda_{2} y_{k}}$. The above equation closely follows the data model shown in the ACMP method, however, since the result for the Fourier transform has 
a frequency-varying amplitude, the ACMP method cannot be applied to find the locations $\left(x_{k}, y_{k}\right)$. Having said that, by setting $m$ and $n$ indices to zero separately, we will end up with two equations in power-sum series form, which means that annihilating filter method can be used to retrieve the parameters $x_{k}$ and $y_{k}$ separately. We already know that such a method has the problem of finding the correct pairings between the $x_{k}$ and $y_{k}$ coordinates, however, with the use of Radon transform and the projection-slice theorem [11] we can retrieve the locations of the vertices of bi-level polygons from their moments. Projectionslice theorem states that the Fourier transform function $G(u, v)$ evaluated along a line passing through the origin at an angle $\theta$, is identical to the one dimensional Fourier transform of the Radon projection $R_{g}(t, \theta)$. In mathematical form:

$$
G(\omega \cos (\theta), \omega \sin (\theta))=\hat{R}_{g}(\omega, \theta) .
$$

In our set-up, $\theta=\tan ^{-1}\left(\frac{n}{m}\right)$ with $m$ and $n$ being the indices of the moments and $\omega=\sqrt{\left(m^{2}+n^{2}\right)}$. With the help of this mapping, we can transform the Fourier coefficients of bi-level polygons, obtained from E-spline sampling kernel (see equation (4)), to the Radon domain as follows:

$$
\hat{R}_{g}(\omega, \theta)=\tau_{\omega \cos (\theta), \omega \sin (\theta)}=\sum_{k=1}^{K} a_{k} \times e^{\alpha_{\omega \cos (\theta)} x_{k}+\beta_{\omega \sin (\theta)} y_{k}} \times \omega^{-2},
$$

where $a_{k}=\frac{p_{k-1}-p_{k}}{\left(\cos (\theta)+p_{k-1} \sin (\theta)\right)\left(\cos (\theta)+p_{k} \sin (\theta)\right)}$.

Let us introduce $S(\omega, \theta)=\tau_{\omega \cos (\theta), \omega \sin (\theta)} \times \omega^{2}$ to present the new mapped equation. The above equation can now be rewritten as:

$$
S(\omega, \theta)=\sum_{k=1}^{K} a_{k} \times e^{\alpha_{\omega \cos (\theta)} x_{k}+\beta_{\omega \sin (\theta)} y_{k}} .
$$

At $\omega=0, S(\omega, \theta)=0$ so the minimum required spline order can be decreased by 1 as the first data sample is always zero. Since the angle $\theta$ is fixed for a given projection, then the mapped equation at different projections has a power-sum series form:

$$
S(\omega, \theta)=\sum_{k=1}^{K} \widehat{a_{k}} e^{\omega z_{k}}=\sum_{k=1}^{K} a_{k} u_{k}^{\omega}
$$

where $\widehat{a_{k}}=a_{k} e^{\alpha_{0} x_{k}} e^{\beta_{0} y_{k}}, z_{k}=x_{k} \cos (\theta) \lambda+y_{k} \sin (\theta) \lambda$ and $u_{k}=e^{z_{k}}$. By using annihilating filter method we can find all the parameters $z_{k}$ for each projection. For example, by setting $m=0$ and $n=0$ we have the projections at $\theta=0$ and $\theta=90$ degrees respectively, likewise if $m=n$ then we have the projection at 45 degrees. Further angles can be obtained by choosing different patterns such as $m=2 n$ or $n=2 m$. Each projection, as explained above, will result in a power-sum series form and annihilating filter method can be used to retrieve the 


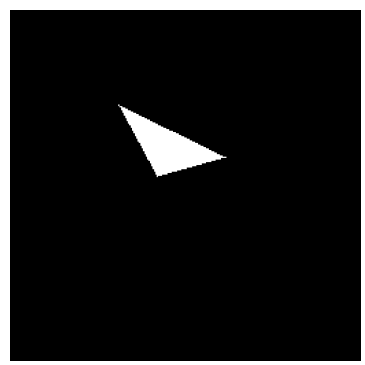

(a)

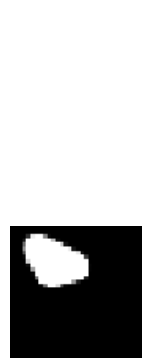

(b)

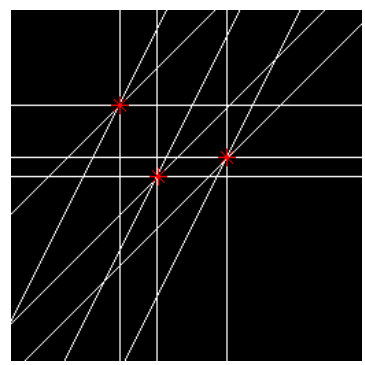

(c)

Figure 4: (a) A 3-sided polygon in a frame size of 256x256 (b) The 32x32 samples of the input signal (c) The reconstructed vertices with $3+1=4$ back-projections, the crosses are the actual vertices of the polygon. [Not to scale]

parameters $z_{k}$ which correspond to the sums of the vertices of the polygon in different directions. By back-projecting the parameters $z_{k}$ according to their $\theta$ we are able to retrieve some information about the polygon's vertices. As any $K$-sided convex and bi-level polygon is completely specified by the location of its $K$ vertices, it is known [16] that $K+1$ projections will entirely specify the vertices of the bi-level polygon, that is, points that have $K+1$ line intersections from the back-projections correspond to the $K$ vertices.

To reconstruct a set of $K$ Diracs from its samples, we need at least $2 K$ data points, which means a minimum spline order of $2 K$ is required. For bi-level polygons however, as the first data sample is always zero, a minimum spline order of $2 K-1$ at each projection angle is required. Thus, the minimum spline order required for a perfect reconstruction of a given $K$-sided bi-level polygon is $p .(2 K-1)$ where $p$ is the number needed in order to produce at least $K+1$ projections. The value of $p$ can be found by inspection but it can be shown that $p$ is $\mathcal{O}(K)$, thus, the order of the spline is $\mathcal{O}\left(K^{2}\right)$ along both directions. Figure 4 shows an example of the sampling process where the input signal, corresponding samples and the reconstructed signal are shown. We can now summarize the above discussion with the following proposition:

Proposition II - A $K$-sided bi-level polygon is perfectly reconstructed from the samples $s_{j, k}=\left\langle g(x, y), \phi\left(\frac{x}{T_{x}}-j, \frac{y}{T_{y}}-k\right)\right\rangle$ with a minimum number of samples $N=2 K L$ along both the Cartesian axes $x$ and $y$, provided that the sampling kernel $\phi(x, y)$ with support $L$, can reproduce exponentials with an order $p(2 K-$ 1 ) along both directions, where $p$ is the number required in order to produce at least $K+1$ projections.

The 2-D order of the spline required is $\mathcal{O}\left(K^{2}\right) \times \mathcal{O}\left(K^{2}\right)$ and this suggests that as the complexity of the signal increases, a higher sampling rate will be required. In the next section we show how this can be avoided by using a multichannel acquisition system. 


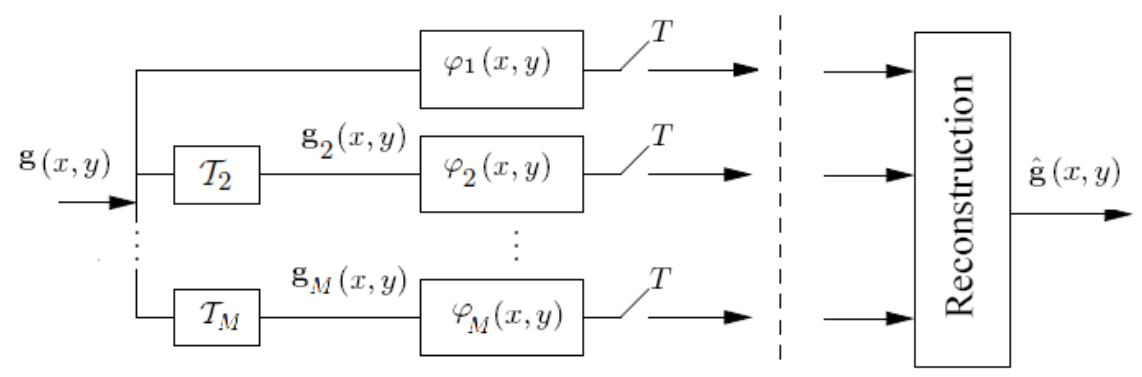

Figure 5: A multichannel sampling setup for 2-D FRI signals. The bank of Espline filters $\varphi_{1}, \varphi_{2}, \ldots, \varphi_{M}$ receive different geometrically transformed versions of the original signal $g(x, y)$. Here, the unknown transformation parameters are denoted by $\mathcal{T}_{2}, \ldots, \mathcal{T}_{M}$.

\section{Multichannel Sampling of Parametric Signals}

In this section we investigate the scenario of multichannel sampling of 2-D parametric signals. Multichannel sampling was first proposed by Papoulis in the context of bandlimited signals [19] and extended by Unser et al. [24] for signals lying in shift-invariant subspaces. A further extension related to union of shift-invariant subspaces has been recently considered in [9]. The multichannel sampling of FRI signals has been considered in [13], [18], [20] and [4]. In [20] Seelamantula and Unser, by using simple RC filters, propose a simple acquisition and reconstruction method within the framework of multichannel sampling, where 1-D FRI signals such as an infinite stream of nonuniformly-spaced Dirac impulses and piecewise-constant signals can be sampled and perfectly reconstructed. In [13] Kusuma and Goyal proposed new ways of sampling 1-D Dirac impulses using a bank of integrators or B-splines. Their proposed scheme is closely related to previously known cases but provides a successive approximation property, which could be useful for detecting undermodelling when the number of Dirac impulses are unknown. In [4] Baboulaz and Dragotti use a multichannel sampling setup for sampling FRI signals and utilize that for image registration based on continuous moments.

A model of the multichannel system accepting multidimensional signals is shown in Figure 5 where the bank of E-spline filters $\varphi_{1}, \varphi_{2}, \ldots, \varphi_{M}$ receive different geometrically transformed versions of the original signal $g(x, y)$. Here, the unknown transformation parameters are denoted by $\mathcal{T}_{2}, \ldots, \mathcal{T}_{M}$.

We have looked at the case of multichannel sampling of a stream 1-D Diracs in [3] using exponential splines, with unknown gains and delays introduced within the channel and illustrated that by synchronizing the different channels of the proposed multichannel sampling setup, one can estimate the unknown delays and gains, regardless of the input FRI signal. We will now consider the 
multichannel sampling of multidimensional signals. Let us first assume that our input signal is a set of $K 2$-D Diracs and the geometric transformations in each channel is restricted to 2-D translations $\left(\Delta x_{i}, \Delta y_{i}\right)$, only. The signal $g(x, y)$ is acquired with a multichannel sampling system shown in Figure 5 with $M$ channels. The sampling kernel in each channel is able to reproduce exponentials and for simplicity we assume that each kernel is an E-spline of order $P \times Q$. The samples of the i-th channel, with $i=2,3, \ldots, M$, are given by:

$$
s_{j, k}^{i}=\left\langle g\left(x-\Delta x_{i}, y-\Delta y_{i}\right), \varphi\left(\frac{x}{T_{x}}-j, \frac{y}{T_{y}}-k\right)\right\rangle,
$$

with $\left(\Delta x_{1}, \Delta y_{1}\right)=(0,0)$. Our goal is to have a reconstruction technique that can perfectly retrieve the unknown translation parameters as well as the input signal. For the sake of clarity, let us assume that $M=2$ and that:

$$
\begin{gathered}
\hat{\varphi}_{1}\left(\omega_{1}, \omega_{2}\right)=\prod_{m=0}^{P} \prod_{n=0}^{2 Q-1}\left(\frac{1-e^{\alpha_{m}-j \omega_{1}}}{j \omega_{1}-\alpha_{m}}\right)\left(\frac{1-e^{\beta_{n}-j \omega_{2}}}{j \omega_{2}-\beta_{n}}\right) \\
\hat{\varphi}_{2}\left(\omega_{1}, \omega_{2}\right)=\prod_{m=P}^{2 P-1} \prod_{n=0}^{2 Q-1}\left(\frac{1-e^{\alpha_{m}-j \omega_{1}}}{j \omega_{1}-\alpha_{m}}\right)\left(\frac{1-e^{\beta_{n}-j \omega_{2}}}{j \omega_{2}-\beta_{n}}\right) .
\end{gathered}
$$

We have set one parameter to be common between the exponents of E-spline in one direction, specifically, both kernels can reproduce the exponentials $e^{\alpha_{P} x} e^{\beta_{n} y}$ for $n=0,1, \ldots, 2 Q-1$. Using the samples, we can obtain the exponential moments $\tau_{m, n}^{i}$ for both channels as indicated in Equations 5-7. More precisely we have:

$$
\tau_{m, n}^{1}=\int_{-\infty}^{\infty} \int_{-\infty}^{\infty} g(x, y) e^{\alpha_{m} x} e^{\beta_{n} y} d x d y
$$

where $m=0,1, \ldots, P$ and $n=0,1, \ldots, 2 Q-1$ and,

$$
\tau_{m, n}^{2}=\int_{-\infty}^{\infty} \int_{-\infty}^{\infty} g\left(x-\Delta x_{2}, y-\Delta y_{2}\right) e^{\alpha_{m} x} e^{\beta_{n} y} d x d y=\tau_{m, n}^{1} e^{\alpha_{m} \Delta x_{2}} e^{\beta_{n} \Delta y_{2}}
$$

where $m=P, P+1, \ldots, 2 P-1$ and $n=0,1, \ldots, 2 Q-1$. Given the above equations, by taking logarithms we will obtain a system of simple linear equations which we can solve for the translation parameters $\Delta x_{2}$ and $\Delta y_{2}$, that is:

$$
\left(\begin{array}{l}
\Delta x_{2} \\
\Delta y_{2}
\end{array}\right)=\left(\begin{array}{ll}
\alpha_{P} & \beta_{0} \\
\alpha_{P} & \beta_{1}
\end{array}\right)^{-1}\left(\begin{array}{l}
\ln \left(\frac{\tau_{P, 0}^{2}}{\tau_{P, 0}^{1}}\right) \\
\ln \left(\frac{\tau_{P, 1}^{2}}{\tau_{P, 1}^{1}}\right)
\end{array}\right)
$$

This reveals that, independently of $g(x, y)$ it is possible to synchronize the two channels exactly from the obtained measurements. Given the exact translation 
parameters, we can now estimate the exponential moments $\tau_{m, n}^{1}$, with $m=$ $P, P+1, \ldots, 2 P-1$ from $\tau_{m, n}^{2}$ as follows:

$$
\tau_{m, n}^{1}=\tau_{m, n}^{2} e^{-\alpha_{m} \Delta x_{2}} e^{-\beta_{n} \Delta y_{2}},
$$

where $m=P, P+1, \ldots, 2 P-1$ and $n=0,1, \ldots, 2 Q-1$.

For the case of set of 2-D Diracs for example, it follows that $P \geq K$ so that perfect recovery of the signal $g(x, y)$ is possible from the moments $\tau_{m, n}^{1}$ for $m=0,1, \ldots, 2 P-1$ and $n=0,1, \ldots, 2 Q-1$. The advantage of the set-up above is that we now require splines of lower order, i.e. $P \geq K$ rather than $P \geq 2 K-1$, and this leads to shorter kernels in one of the dimensions. This indicates that we can either sample signals with a higher complexity or alternatively for the same signal we can almost halve the sampling rate in one of the dimensions. The extension to the case of $M$ channels is also straightforward. By designing each sampling kernel so that pairs of channels have one parameter in common in one direction, it is possible to synchronize the channels and then reconstruct $g(x, y)$. This means that by using an M-channel system we can either sample the same FRI signals with a reduced sampling rate proportional to $\sim 1 / T M$ or sample signals with a much higher complexity.

The above method can be applied to any 2-D FRI signals as long as the transformation parameters are restricted to 2-D translations. However, one can not estimate more complicated geometric transformations such as rotation and scaling with exponential reproducing kernels. This is because introducing such parameters would result in a non-linear relationship between the exponential moments of the different signals and therefore our introduced method above cannot be applied. This problem can be solved with the use of geometric moments $[10,15,27]$ which can be obtained by a polynomial reproducing kernel such as B-splines. Let us assume that the input signal is a $K$-sided bi-level polygon with scaling, rotation and translation introduced within different channels. It is known that the parameters of such transformations can be estimated from the geometric moments up to order 3 from the two signals. Therefore, an alternative sampling kernel is required which could reproduce polynomials for the calculation of the transformation parameters, and exponentials for sampling and perfectly reconstructing the input signal. E-splines support such feature as they are a generalized version of B-splines [23], thus, a combination of polynomials and exponentials from E-splines can be reproduced. The Fourier transform of the corresponding generalized E-spline function will then be as follows:

$\hat{\beta}_{\vec{\alpha}, \vec{\beta}}\left(\omega_{1}, \omega_{2}\right)=\left(\frac{1-e^{-j \omega_{1}}}{j \omega_{1}}\right)^{3}\left(\frac{1-e^{-j \omega_{2}}}{j \omega_{2}}\right)^{3} \prod_{m=0}^{M} \prod_{n=0}^{N}\left(\frac{1-e^{\alpha_{m}-j \omega_{1}}}{j \omega_{1}-\alpha_{m}}\right)\left(\frac{1-e^{\beta_{n}-j \omega_{2}}}{j \omega_{2}-\beta_{n}}\right)$.

The produced spline is of order $(M+4) \times(N+4)$, has compact support and can reproduce polynomials up to order 3 along both directions and can 
reproduce any exponential up to order $(M+1) \times(N+1)$. Now that the unknown parameters are estimated from the generalized sampling kernel, we can synchronize the channels in our multichannel set-up and perfectly retrieve the bi-level polygon signal as follows: It was already shown in this paper that with $K+1$ projections, a $K$-sided bi-level polygon can be sampled and perfectly reconstructed. Moreover, it is known that Radon projection at angle $\phi$ of an image rotated with an angle $\theta$ with respect to its reference image, is equivalent of the reference image at the angle $\phi+\theta$. Therefore, the $K+1$ projections required to perfectly reconstruct the input bi-level polygon could be obtained from the different channels and this leads to shorter kernels and thus less samples will be required from each channel.

The steps given below shows the procedures required to sample bi-level polygons in the proposed multichannel framework, with scaling, rotation and translation introduced within different channels. For the sake of clarity, we have assumed $M=2$ :

1. Choose the sampling kernel on both channels to be of order $N_{1}+3$ and $N_{2}+3$ respectively. Here, the number 3 corresponds to the spline order which is required for estimating the transformation parameters and the numbers $N_{1}$ and $N_{2}$ correspond to the 2-D spline order required to produce at least $P$ and $Q$ projections respectively, with $P+Q \geq K+1$.

2. Estimate the transformation parameters between the two channels from their corresponding geometric moments.

3. Obtain the $P$ projections at the angles $\phi_{1}, \phi_{2}, \ldots, \phi_{P}$ from the first channel and back-project them, as described in Section 3.

4. Obtain the $Q$ projections at the angles $\psi_{1}, \psi_{2}, \ldots, \psi_{Q}$ from the second channel.

5. Un-do the already estimated scaling and translations parameters on the projections from the second channel and obtain the projections of the input signal at the angles $\theta+\psi_{1}, \theta+\psi_{2}, \ldots, \theta+\psi_{Q}$ where $\theta$ is the estimated rotation parameter.

6. Back-project the projections in 3 and 5 to achieve perfect reconstruction of the input bi-level polygon.

As an example, in order to achieve perfect reconstruction for a 4-sided bilevel polygon, a 2-D E-spline order of 14 along both directions is required to produce 5 projections at the angles $0,45,90, \tan ^{-1}(2)$ and $\tan ^{-1}\left(\frac{1}{2}\right)$. With 2-D E-spline order of 8 however we can produce 3 projections at the angles $0,45,90$ on the reference signal, and a 2-D E-spline order of 8 on the second signal would give 3 projections for the reference signal at the angles $\theta, 45+\theta, 90+\theta$. Assuming 


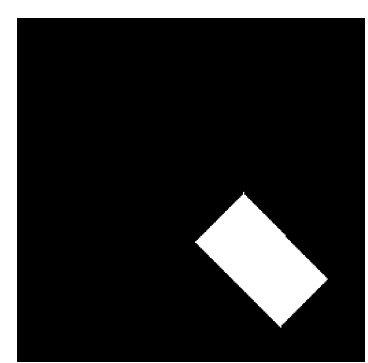

(a)

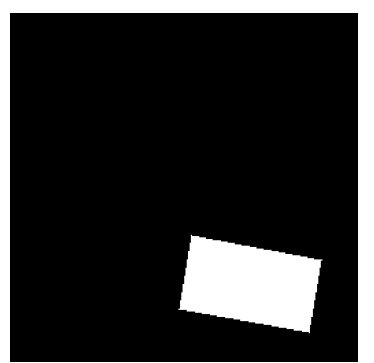

(b)

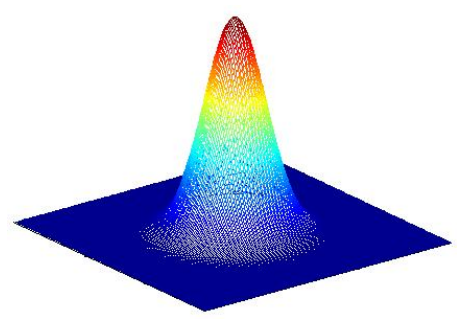

(c)

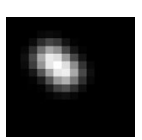

(d)

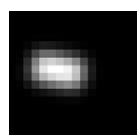

(e)

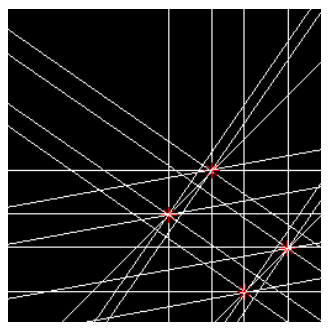

(f)

Figure 6: Multichannel sampling of bi-level polygons using E-spline sampling kernels. (a) The reference signal in a frame data size of $256 \times 256$. (b) The translated $(\triangle x=-100, \triangle y=150)$, rotated $(\theta=35)$ and scaled $(a=1.1)$ version of the reference signal. (c) 2-D generalized E-spline of order 11. (e) \& (d) The $16 \times 16$ samples of both signals. (f) The reconstructed vertices of the reference signal with 6 back-projections, the crosses are the actual vertices of the polygon. [Not to scale].

$\theta$ is not zero, with an spline order of $8+3=11$ on each channel we have enough projections to perfectly reconstruct the input signal. Figure 6 shows an example where the input signal, its translated, rotated and scaled version, their corresponding samples, the E-spline sampling kernel, and the reconstructed input signal are all shown.

\section{Conclusion}

In this paper we showed that with the use of ACMP method, projection-slice theorem and Radon projections, multidimensional parametric signals such as set of 2-D Diracs and bi-level polygons can be sampled and perfectly reconstructed using exponential splines as the sampling kernel. For the case of multichannel sampling scenario, assuming that the geometric transformations are restricted to 2-D translations only, we showed that the different channels can be synchronized regardless of the input FRI signal. For the case of more complicated transforms such as scaling and rotation, we illustrated that with the use of Radon projec- 
tions, one can separate the projections needed between the different channels, to perfectly retrieve the input signal in the multichannel set-up.

\section{References}

[1] H. Akhondi Asl and P.L. Dragotti, Multichannel sampling of translated, rotated and scaled bilevel polygons using exponential splines, Proc. of the 8th international conference on Sampling Theory and Applications, Marseille, France, May 2009.

[2] H. Akhondi Asl and P.L. Dragotti, Single and multichannel sampling of bilevel polygons using exponential splines, Proc. of IEEE Int. Conf. on Acoustic, Speech and Signal Processing, 3349-3352, Taipei, Taiwan, May 2009.

[3] H. Akhondi Asl, P.L. Dragotti, and L. Baboulaz, Multichannel sampling of signals with finite rate of innovation, IEEE Signal Processing Letter, 17(8), 762-765, August 2010.

[4] L. Baboulaz and P.L. Dragotti, Exact feature extraction using finite rate of innovation principles with an application to image super-resolution, IEEE Trans. Image Processing, 18(2), 281-298, Feb. 2009.

[5] J. Berent, P.L. Dragotti, and T. Blu, Sampling piecewise sinusoidal signals with finite rate of innovation methods, IEEE Trans. Signal Processing, 58(2), 613-625, February 2010.

[6] E. Candès, J. Romberg, and T. Tao, Robust uncertainty principle: Exact signal reconstruction from highly incomplete frequency information, IEEE Trans. Information Theory, 52(2), 489-509, February 2006.

[7] D.L. Donoho, Compressed sensing, IEEE Trans. Information Theory, 52(4), 1289-1306, April 2006.

[8] P.L. Dragotti, M. Vetterli, and T. Blu, Sampling moments and reconstructing signals of finite rate of innovation: Shannon meets Strang-Fix. IEEE Trans. Signal Processing, 55(5), 1741-1757, May 2007.

[9] Y. Eldar, Compressed sensing of analog signals in shift-invariant spaces, IEEE Trans. Signal Processing, 57(8), 2986-2997, August 2009.

[10] J. Flusser and T. Suk, A moment-based approach to registration of images with affine geometric distortion, IEEE Trans. Geoscience and Remote Sensing, 32(2), 382-387, March 1994. 
[11] G.T. Herman, Image Reconstruction from Projections: The Fundamentals of Computerized Tomography, Academic Press, New York, 1980.

[12] Y. Huy, Estimating two-dimensional frequencies by matrix enhancement and matrix pencil, IEEE Trans. Signal Processing, 40(9), 2267-2280, Sep. 1992.

[13] J. Kusuma and V.K. Goyal, On the accuracy and resolution of powersumbased sampling methods, IEEE Trans. Signal Processing, 57(1), 182-193, January 2009.

[14] S.W. Lee and R. Mittra, Fourier transform of a polygonal shape function and its application in electromagnetics, IEEE Trans. Antennas and Propagation, 31(1), 99-103, 1983.

[15] H. Li, B. S. Manjunath and S. K. Mitra, A contour-based approach to multisensor image registration, IEEE Trans. on Image Processing, 4(2), 320-334, March 1995.

[16] I. Maravic and M. Vetterli, A sampling theorem for the radon transform of finite complexity objects, In Proc. of IEEE Int. Conf. on Acoustic, Speech and Signal Processing, volume 2, 1197-1200, Orlando, Florida (USA), May 2002 .

[17] I. Maravic and M. Vetterli, Exact sampling results for some classes of parametric non-bandlimited 2-d signals, IEEE Trans. Signal Processing, 52(1), 175-189, 2004.

[18] H. Olkkonen and J.T. Olkkonen, Measurement and reconstruction of impulse train by parallel exponential filter, IEEE Signal Processing Letter, 15, 241-244, February 2008.

[19] A Papoulis, Generalized sampling expansions, IEEE Trans. on Circuits Syst., 24, 652-654, 1977.

[20] C.S. Seelamantula and M. Unser, A generalized sampling method for finiterate-of-innovation-signal reconstruction, IEEE Signal Processing Letter, 15, 813-816, 2008.

[21] P. Shukla and P.L. Dragotti, Sampling schemes for multidimensional signals with finite rate of innovation, IEEE Trans. Signal Processing, 55, 3670$3686,2007$.

[22] P. Stoica and R. Moses, Spectral Analysis of Signals, Prentice Hall, April 2005. 
[23] M. Unser and T. Blu, Cardinal Exponential Splines: Part I-theory and filtering algorithms, IEEE Trans. Signal Processing, 53(4), 1425-1438, April 2005.

[24] M. Unser and J. Zerubia, A generalized sampling theory without bandlimiting constraints, IEEE Trans. Circuits Syst. II, 45(8), 959-969, August 1998.

[25] F. Vanpoucke, M. Moonen, and Y. Berthoumieu, Efficient subspace algorithm for 2-d harmonic retrieval, In Proc. of IEEE Int. Conf. on Acoustic, Speech and Signal Processing, volume 4, 461-464, Toulouse (France), April 1994.

[26] M. Vetterli, P. Marziliano, and T. Blu, Sampling signals with finite rate of innovation, IEEE Trans. Signal Processing, 50(6), 1417-1428, June 2002.

[27] Z. Yang and F. Cohen, Cross-weighted moments and affine invariants for image registration and matching, IEEE Trans. Pattern Analysis and Machine Iintelligence, 21(8), 804-814, August 1999.

\section{Appendix A: Annihilating Filter Method}

In this Appendix we provide a brief review of the annihilating filter method. Consider a power-sum series in the form:

$$
\tau_{m}=\sum_{k=1}^{K} a_{k} u_{k}^{m}, \quad m=1,2, \ldots, M .
$$

Our aim is to retrieve the set of unknown parameters $u_{k}$ and $a_{k}$ from the measurements $\tau_{m}$. Let us define a filter $h_{m}$ with $m=0,1, \ldots, K$, such that the locations $u_{k}$ are the roots of the filter. The z-transform of such a filter is:

$$
H(z)=\sum_{m=0}^{K} h_{m} z^{-m}=\prod_{k=1}^{K}\left(1-u_{k} z^{-1}\right) .
$$

The signal $\tau_{m}$ convolved with the filter defined above, results in:

$$
\begin{aligned}
h_{m} * \tau_{m} & =\sum_{i=0}^{K} h_{i} \tau_{m-i} \\
& =\sum_{i=0}^{K} \sum_{k=1}^{K} a_{k} h_{i} u_{k}^{m-i} \\
& =\sum_{k=1}^{K} a_{k} u_{k}^{m} \underbrace{\sum_{i=0}^{K} h_{i} u_{k}^{-i}}_{=0}=0,
\end{aligned}
$$


The under-braced term in the set of equations above equals to zero, as $H\left(u_{k}\right)=$ 0, thus:

$$
h_{m} * \tau_{m}=0 .
$$

The filter $H(z)$ is called the annihilating filter as it annihilates the signal $\tau_{m}$. The zeros of such a filter uniquely define the distinct locations $u_{k}$. Moreover, the convolution equation can be written in the matrix form as follows:

$$
\left[\begin{array}{cccc}
\tau_{K} & \tau_{K-1} & \cdots & \tau_{0} \\
\tau_{K+1} & \tau_{K} & \cdots & \tau_{1} \\
\vdots & \vdots & \ddots & \vdots \\
\tau_{N} & \tau_{N-1} & \cdots & \tau_{N-K}
\end{array}\right] \times\left[\begin{array}{c}
h(0) \\
h(1) \\
\vdots \\
h(K)
\end{array}\right]=0
$$

where $N \geq 2 K-1$ as at least $2 K$ consecutive values of $\tau_{m}$ are required in order to solve the matrix equation shown above. Since $h(0)=1$, the above system can be written as follows:

$$
\left[\begin{array}{cccc}
\tau_{K-1} & \tau_{K-2} & \cdots & \tau_{0} \\
\tau_{K} & \tau_{K-1} & \cdots & \tau_{1} \\
\vdots & \vdots & \ddots & \vdots \\
\tau_{N-1} & \tau_{N-2} & \cdots & \tau_{N-K}
\end{array}\right] \times\left[\begin{array}{c}
h(1) \\
h(2) \\
\vdots \\
h(K)
\end{array}\right]=-\left[\begin{array}{c}
\tau_{K} \\
\tau_{K+1} \\
\vdots \\
\tau_{N}
\end{array}\right]
$$

where by taking the inverse of the first matrix we can solve for the coefficients $h_{m}$. Given the filter coefficients, the parameters $u_{k}$ are found by taking the roots of the filter. The system of equations above gives a unique solution for $u_{k}$ since the filter coefficients $h_{m}$ are unique for a given signal (if there are multiple poles, for example $u_{k-1}=u_{k}$, then the most-left matrix above will be rank deficient and the filter coefficients $h_{m}$ will not be unique). After finding the locations $u_{k}$, we are able to find the weights $a_{k}$ from the power-sum series formula given in equation (43). By expanding the equation and writing it in the matrix form, we obtain:

$$
\left[\begin{array}{cccc}
1 & 1 & \cdots & 1 \\
u_{1} & u_{2} & \cdots & u_{K} \\
u_{1}^{2} & u_{2}^{2} & \cdots & u_{K}^{2} \\
\vdots & \vdots & \ddots & \vdots \\
u_{1}^{K-1} & u_{2}^{K-1} & \cdots & u_{K}^{K-1}
\end{array}\right] \times\left[\begin{array}{c}
a_{1} \\
a_{2} \\
\vdots \\
a_{K}
\end{array}\right]=\left[\begin{array}{c}
\tau_{0} \\
\tau_{1} \\
\vdots \\
\tau_{K-1}
\end{array}\right] .
$$

The above system of equations is a Vandermonde system and leads to a unique solution for the amplitudes $a_{k}$ since the $u_{k}$ are distinct. 